\title{
Expert consensus on practical aspects in the treatment of chronic urticaria
}

\author{
Andrea Bauer · Heinrich Dickel · Thilo Jakob · Andreas Kleinheinz · Undine Lippert · Martin Metz • \\ Sibylle Schliemann · Uwe Schwichtenberg • Petra Staubach · Eva Valesky · Nicola Wagner • Bettina Wedi · \\ Marcus Maurer
}

Received: 18 December 2020 / Accepted: 30 December 2020 / Published online: 24 February 2021

(C) The Author(s) 2021

\begin{abstract}
Background Chronic urticaria (CU) is a common disease which represents a considerable burden for many patients. The current urticaria guideline describes the evidence-based diagnosis and treatment of CU. In addition, however, questions often arise in everyday practice that are not addressed by the guideline.
\end{abstract}

Supplementary Information The online version of this article (https://doi.org/10.1007/s40629-021-00162-w) contains supplementary material, which is available to authorized users.

\section{Prof. Dr. med. A. Bauer}

University Hospital Carl Gustav Carus, Department of Dermatology, University Allergy Center, Urticaria Center of Reference and Excellence (UCARE), Technical University, Dresden, Germany

PD Dr. med. H. Dickel

Department of Dermatology, Venereology and Allergology, St. Josef Hospital, Ruhr University Bochum, Bochum, Germany

Prof. Dr. med. T. Jakob

University Medical Center Giessen (UKGM), Department of Dermatology and Allergy, Justus-Liebig-University Giessen, Giessen, Germany

Dr. med. A. Kleinheinz

Department of Dermatology, Urticaria Center of Reference and Excellence (UCARE), Elbe Medical Centre, Buxtehude, Germany

PD Dr. med. U. Lippert

Department of Dermatology and Allergology, Urticaria Center of Reference and Excellence (UCARE), University Medical Center Göttingen, Göttingen, Germany

Prof. Dr. med. M. Metz · Prof. Dr. med. M. Maurer $(\bowtie)$ Department of Dermatology and Allergy, Urticaria Center of Reference and Excellence (UCARE), Charité-Universitätsmedizin Berlin, Charitéplatz 1, 10117 Berlin, Germany marcus.maurer@charite.de
Methods In May 2020, a digital meeting with German urticaria experts was held, in which practical aspects of CU treatment were discussed and supporting aids for everyday clinical treatment formulated. The resulting advice in this document focus on practical questions and the available literature and experiences of the participants.

Results The diagnosis of CU can be made in a short time by means of a thorough anamnesis, a physical examination, and a basic laboratory chemical diag-

PD Dr. med. S. Schliemann

Department of Dermatology, Urticaria Center of Reference and Excellence (UCARE), University Hospital Jena, Jena, Germany

Dr. med. U. Schwichtenberg

Derma-nord dermatologists Dr. Schwichtenberg, Bremen, Germany

Prof. Dr. med. P. Staubach

Department of Dermatology, Urticaria Center of Reference and Excellence (UCARE), University Medical Center Mainz, Mainz, Germany

PD Dr. med. E. Valesky

University Hospital Frankfurt, Department of Dermatology, Venerology and Allergology, Goethe University, Frankfurt, Germany

Dr. med. N. Wagner

Department of Dermatology, Urticaria Center of Reference and Excellence (UCARE), University Medical Center Erlangen, Erlangen, Germany

Prof. Dr. med. B. Wedi

Department of Dermatology and Allergy, Urticaria Center of Reference and Excellence (UCARE), Comprehensive Allergy Center, Hannover Medical School, Hannover, Germany 
nosis. For this purpose, practical recommendations for everyday practice are given in this paper. An extended diagnosis is only indicated in a few cases and should always be carried out in parallel with an effective therapy. In general, CU should always be treated in the same way, regardless of whether wheals, angioedema or both occur. Symptomatic therapy should be carried out according to the treatment steps recommended by the guidelines. This publication provides practical advice on issues in everyday practice, such as the procedure in the current coronavirus disease 2019 (COVID-19) pandemic, the cardiac risk under higher dosed $\mathrm{H} 1$ antihistamines, the self-administration of omalizumab as well as vaccination under omalizumab therapy. In addition to treatment recommendations, topics such as documentation in the practice and family planning with urticaria will be discussed.

Discussion These supporting treatment recommendations serve as an addendum to the current CU guideline and provide support in dealing with CU patients in everyday practice. The aim is to ensure that patients suffering from CU achieve complete freedom of symptoms with the help of an optimal therapy.

Keywords Treatment recommendations $\cdot \mathrm{H} 1$ antihistamines · Omalizumab · Diagnostics · Therapy

\section{Abbreviations}

AAE Acquired angioedema due to C1-in-

AAS Angioedema activity score hibitor deficiency

ACE Angiotensin-converting enzyme

ACE-Inh Angiotensin-converting enzyme inhibitor

AE Angioedema

AE-QoL Angioedema Quality of Life Questionnaire

AID Auto-inflammatory disease

BAC Blood alcohol content

BSG Blood sedimentation rate

CAPS Cryopyrin-associated periodic syndrome

CholUAS CIndU-specific activity score for cholinergic urticaria

CIndU Chronic inducible urticaria

ColdUAS CIndU-specific activity score for cold urticaria

CRP C-reactive protein

CSA Ciclosporin A

csU Chronic spontaneous urticaria

CU Chronic urticaria

CU-Q2oL Chronic Urticaria Quality of Life Questionnaire

CURE Chronic Urticaria Registry

HAE Hereditary angioedema

NSAID Non-steroidal anti-inflammatory drug

SDAS Activity score for symptomatic dermographism

UAS Urticaria activity score

UCT Urticaria control test

\section{Introduction}

Chronic urticaria (CU; duration of symptoms longer than 6 weeks) is a common disease associated with a considerable burden for many patients, as itching, wheals and angioedema are often not sufficiently controlled. The disease, which occurs at any age, is divided into chronic spontaneous (i.e. with spontaneous onset of symptoms) urticaria (csU) and chronic inducible (i.e. with symptoms triggered by reproducible specific triggers such as cold or pressure) urticaria (CIndU) [1]. Hybrid types (i.e. symptomatic dermographism plus csU) can also occur. The effects of CU go beyond the physical symptoms and significantly reduce the quality of life of those affected. In one third of patients, CU is associated with depression, anxiety as well as sleep disorders, and patients' performance in everyday life, at school and at work is often significantly impaired [1-3]. Therefore, the treatment goal is to achieve complete freedom of symptoms. For many patients, this goal can be achieved with the currently available treatment options. However, these are frequently not optimally implemented in clinical practices. In many cases, drug treatment in everyday clinical practice does not correspond to the guideline recommendations [4]. Current data from the AWARE study show that, despite therapy, about one third of patients do not achieve adequate disease control even after two years [5]. General practitioners are often the initial contacts. In the case of mild disease courses, $\mathrm{H} 1$ antihistamine treatment is usually sufficient. In the case of therapy resistance, a referral to a specialist should be made for escalation of therapy in accordance with the guidelines, and if necessary, also for further diagnosis. The current urticaria guideline discusses in detail the evidence-based diagnosis and treatment of CU. But beyond that, questions often arise in clinical everyday life. For this reason, a meeting of German urticaria experts was held in May 2020 with the aim of discussing practice-relevant aspects of CU treatment and formulating supporting aids for everyday clinical treatment. These resulting pieces of advice serve as a supplement to the current CU guidelines. They focus on practical issues and are based on the available literature as well as experience of the experts in this field.

\section{Chronic urticaria in times of COVID-19}

To date, there is no evidence that patients with CU have a higher risk of severe COVID-19 due to their disease. According to current knowledge, treatment with $\mathrm{Hl}$ antihistamines and biologicals such as omalizumab does not represent an additional risk factor. Studies even suggest that omalizumab can reduce virus-mediated exacerbations [6, 7], which could possibly be beneficial in case of a SARS-CoV-2 (severe 
acute respiratory syndrome coronavirus type 2) infection [8].

It is important to achieve adequate symptom control of CU, even in times of the COVID-19 (coronavirus disease 2019) pandemic. National and international allergology societies and associations (AeDA, DGAKI, GPA, LGAI, ÖGP, ARIA, EAACI ${ }^{1}$ ) therefore recommend that biological therapy should be continued unchanged in patients without suspected or proven SARS-CoV-2 infection. Patients with a mild/moderate COVID-19 course are also recommended to continue therapy (under risk-benefit assessment and with the patient's consent). In case of severe disease, an extension of the interval or interruption of therapy may be considered [9].

\section{Diagnosis of csU}

The objectives of the diagnostic evaluation of patients with csU comprise the exclusion of other diseases (differential diagnosis), testing for trigger factors relevant to $\operatorname{csU}$ (e.g. taking painkillers), evaluating comorbidities, and determining disease activity (symptoms), impairment of quality of life as well as disease control.

\section{Anamnesis and basic diagnostics}

The diagnosis begins with a detailed anamnesis that should be focused on central information. The guideline, which contains 13 specific questions, can be helpful during this process [10]. It is recommended to use a standardised anamnesis questionnaire. A basic questionnaire (see appendix) or the more detailed patient questionnaires of the Chronic Urticaria Registry (CURE) are suitable for initial and follow-up visits (available at: http://www.urticaria-registry.com/forparticipants.html).

Following the anamnesis (including questions on atopic diathesis and gastrointestinal symptoms), a physical examination and basic laboratory diagnostics should be performed. The basic diagnostic tests include the determination of the blood sedimentation rate (BSG) and/or the C-reactive protein (CRP) and a differential blood count [10].

If the anamnesis provides a specific indication for extended diagnostic tests, an effective guidelinebased therapy with the aim of complete freedom of symptoms should immediately be started in parallel to the initiated examinations.

\footnotetext{
${ }^{1}$ AeDA Ärzteverband Deutscher Allergologen, DGAKI Deutsche Gesellschaft für Allergologie und klinische Immunologie, GPA Gesellschaft für Pädiatrische Allergologie und Umweltmedizin, $L G A I$ Luxemburgische Gesellschaft für Allergologie und Immunologie, ÖGP Österreichische Gesellschaft für Pneumologie, ARIA Allergic Rhinitis and its Impact on Asthma, EAACIEuropäischen Akademie für Allergologie und Klinische Immunologie.
}

\section{Differential diagnostics}

Important differential diagnoses in which wheals and angioedema can also occur (e.g. autoinflammatory diseases) should be considered in the anamnesis by means of specific queries (see points in Infobox 1). If a suspicion arises, further clarification by a specialist is recommended.

In patients with wheals (but no angioedema), urticarial vasculitis and autoinflammatory diseases such as Schnitzler syndrome or cryopyrin-associated periodic syndrome (CAPS) must be excluded. For differential diagnosis, the use of the diagnostic algorithm of the guidelines is recommended (Fig. 1; [10]).

Angioedema are often underdiagnosed in patients with csU. They have a negative impact on quality of life and daily activities [11]. Approximately 10\% of all patients with csU experience angioedema only without wheals. In these csU patients with isolated recurrent angioedema, there is a risk of confusion with bradykinin-mediated forms of angioedema, where wheals are also not present. It is therefore relevant for the initiation of therapy to correctly diagnose and classify angioedema. Bradykinin-mediated angioedema include angiotensin-converting enzyme (ACE)-inhibitor-induced angioedema, sartan-mediated angioedema, and hereditary angioedema (HAE) as well as angioedema due to acquired $\mathrm{Cl}$ inhibitor deficiency. These differential diagnoses can be proven or ruled out by a specific anamnesis, discontinuation of suspected medication (ACE inhibitors) and laboratory tests (determination of the concentration as well as activity of the $\mathrm{Cl}$ esterase inhibitor [C1INH] and the complement factor C4 and possibly C1q). It should be emphasised that csU-associated angioedema rarely lead to swelling of the tongue and only in exceptional cases affect the larynx (e.g. non-steroidal anti-inflammatory drugs [NSAIDs] as a pharmacological trigger). Patients should be informed about this to reduce any anxiety.

In addition, differentiation from other cutaneous/ subcutaneous swellings is essential (see Infobox 2 for examples).

In few cases, a true IgE-mediated food allergy may manifest itself under the clinical picture of urticaria. Diagnosis of this should be carried out by a specialist/allergist. Intermittent occurrence of urticaria and/or accompanying symptoms, such as gastrointestinal problems, may be indicative. This form of food allergy is rarely relevant in patients with CU but is more likely if wheals do not occur daily. Pseudo-allergic reactions (non-IgE-mediated hypersensitivity) to various food ingredients and additives are more frequently observed.

\section{Evaluation of triggering factors}

Since there are variable triggering factors of csU, the medical history is of central importance in identifying 


\section{Infobox 1}

1. Family history of wheals and angioedema

- A positive family history may indicate congenital diseases such as autoinflammatory syndromes and hereditary angioedema (HAE).

2. Timing of disease onset

- Onset in early childhood may indicate congenital conditions such as cryopyrin-associated periodic syndrome (CAPS) or HAE.

3. Occurrence of angioedema without the presence of wheals

- Isolated occurrence of angioedema may indicate bradykinin-mediated angioedema, e.g. HAE or ACE inhibitor/sartan-mediated angioedema.

4. Duration and location of angioedema

- Angioedema that persist for several days, angioedema of the larynx or abdomen or angioedema that do not respond to glucocorticoid therapy may indicate HAE or other bradykinin-mediated angioedema.

5. Duration and consequences of wheals

- Long duration of wheals (usually $>24 \mathrm{~h}$ ) or subsequent haematoma or hyperpigmentation may indicate urticarial vasculitis.

6. Medication

- Taking ACE inhibitor or sartan may indicate ACE inhibitor/sartan-mediated angioedema.

7. Associated symptoms

- Bone/joint pain, signs of inflammation or fever may indicate autoinflammatory syndrome.

8. Occurrence of wheals and/or angioedema depending on specific triggers

- The exclusive occurrence of wheals and/or angioedema due to specific triggers (e.g. skin exposed to cold) indicates chronic inducible urticaria.

9. Previous therapy and response to therapy (including dosage and duration)

- Therapy resistance may indicate autoinflammatory syndrome or bradykinin-mediated angioedema.

10. Previous diagnostic procedures/results

11. Intermittent occurrence of urticaria and/or accompanying symptoms, e.g. gastrointestinal problems

- May indicate an IgE-mediated food allergy.

triggers, but also predictors of disease progression and therapy as well as factors influencing disease activity [10]. We advise against a premature search for infections without anamnestic signs; however, this should be done in cases of prolonged persistence and/or severe suffering.

\section{Evaluation of comorbidities}

In routine diagnostics, frequent comorbidities of csU should be clarified by anamnesis and, if necessary,

\section{Infobox 2}

- Granulomatous inflammation (e.g. cheilitis granulomatosis, genital oedema in Crohn's disease)

- Contact urticaria, acute allergic contact eczema

- Air emphysema (e.g. after fractures, endodontological treatments)

- Tumours

- Oedema in heart failure or chronic venous insufficiency

- Pregnancy oedema and oedema in premenstrual syndrome

further diagnostic tests. Comorbidities can influence the severity of csU, the impairment of quality of life and the response to therapy.

Autoimmune diseases (especially of the thyroid gland) The determination of thyroid hormones and autoantibodies to identify comorbidity (Hashimoto's thyroiditis) is recommended, as patients with csU are more often affected by autoimmune thyroid diseases [12].

\section{Psychiatric comorbidities}

Patients with csU often develop psychiatric comorbidities such as depression and anxiety disorder [13]. For these patients, interdisciplinary cooperation and therapy, e.g. with psychotherapists, is recommended.

\section{CIndU}

CIndU often occurs as a simultaneous disease in csU (about 20\%) and is the most common comorbidity in H1-antihistamine-resistant csU [40]. It should therefore always be examined whether CU patients also suffer from CIndU and vice versa.

\section{Assessment of disease activity, impairment of quality of life and disease control}

In everyday clinical practice, the assessment of the disease burden, defined as disease activity plus quality of life and disease control, supports the decision whether therapy is successful or should be escalated if necessary.

To monitor disease activity, the weekly urticaria activity score (UAS7) [14] and the angioedema activity score (AAS) [15] are used. To calculate the UAS7, wheals and itching are documented and quantified daily by the patient in a diary over seven consecutive days [14]. The occurrence of angioedema is recorded along the same lines with the AAS [15].

It is important to note that the UAS7 is only applicable to csU and not suitable for CIndU. However, CIndU-specific activity scores are available (CholUAS7 for cholinergic urticaria) or in development (ColdUAS 
for cold urticaria, SDAS for symptomatic dermographism).

To assess disease control and therapy success in csU, CIndU, and combinations of both subtypes, the urticaria control test (UCT) is used. With four simple questions, it enables a quick and reliable assessment of the disease situation during the last four weeks. Each of the four UCT questions is answered by the patient, and the resulting scores ( $0-4$ per answer) are added to a total score of $0-16$. The threshold for controlled urticaria is reached at 12 points. Uncontrolled urticaria is indicated if the patient reaches $\leq 11$ points [16].

In addition, regular assessment of the quality of life is useful to estimate treatment success. In addition to the DLQI, two instruments are available for this purpose: the Chronic Urticaria Quality of Life Questionnaire (CU-Q2oL) [17] and the Angioedema Quality of Life Questionnaire (AE-QoL) [18].

\section{Therapy of CU}

The primary and uniform goal of CU treatment is to achieve complete freedom of symptoms (treat the disease until it is gone). The therapeutic approach consists of two aspects:

- Elimination and prevention of relevant triggers and

- Symptomatic drug therapy [10].

\section{Elimination and prevention of relevant triggers}

If a patient has one or more relevant triggers for the occurrence of wheals and/or angioedema (often there are no known triggers), these should be eliminated or avoided if possible. Avoidance almost never results in complete recovery, but it can lead to an improvement of symptoms. Drugs suspected of being triggers should be discontinued or, if necessary, replaced with drugs from another substance class. If csU is triggered by NSAIDs, a switch of analgesics to paracetamol can be attempted. From a differential diagnosis point of view, a NSAID hypersensitivity must be considered.

If physical stimuli trigger the disease, avoidance is desirable, but often not feasible in everyday life. Here it is important to provide patients with knowledge about their illness. In the case of pressure urticaria and symptomatic dermographism, for example, it should be explained that even simple methods (e.g. wider pocket straps, avoidance of tight-fitting clothes and belts) can help to reduce the development of symptoms. As it is not always possible to avoid cold when suffering from cold urticaria, patients should also be offered help in everyday life. Thick, warm clothing, (ski) gloves and warm shoes/ socks are important protective measures against the cold. Unprotected areas such as the face should be treated with a fatty ointment before going outside. In

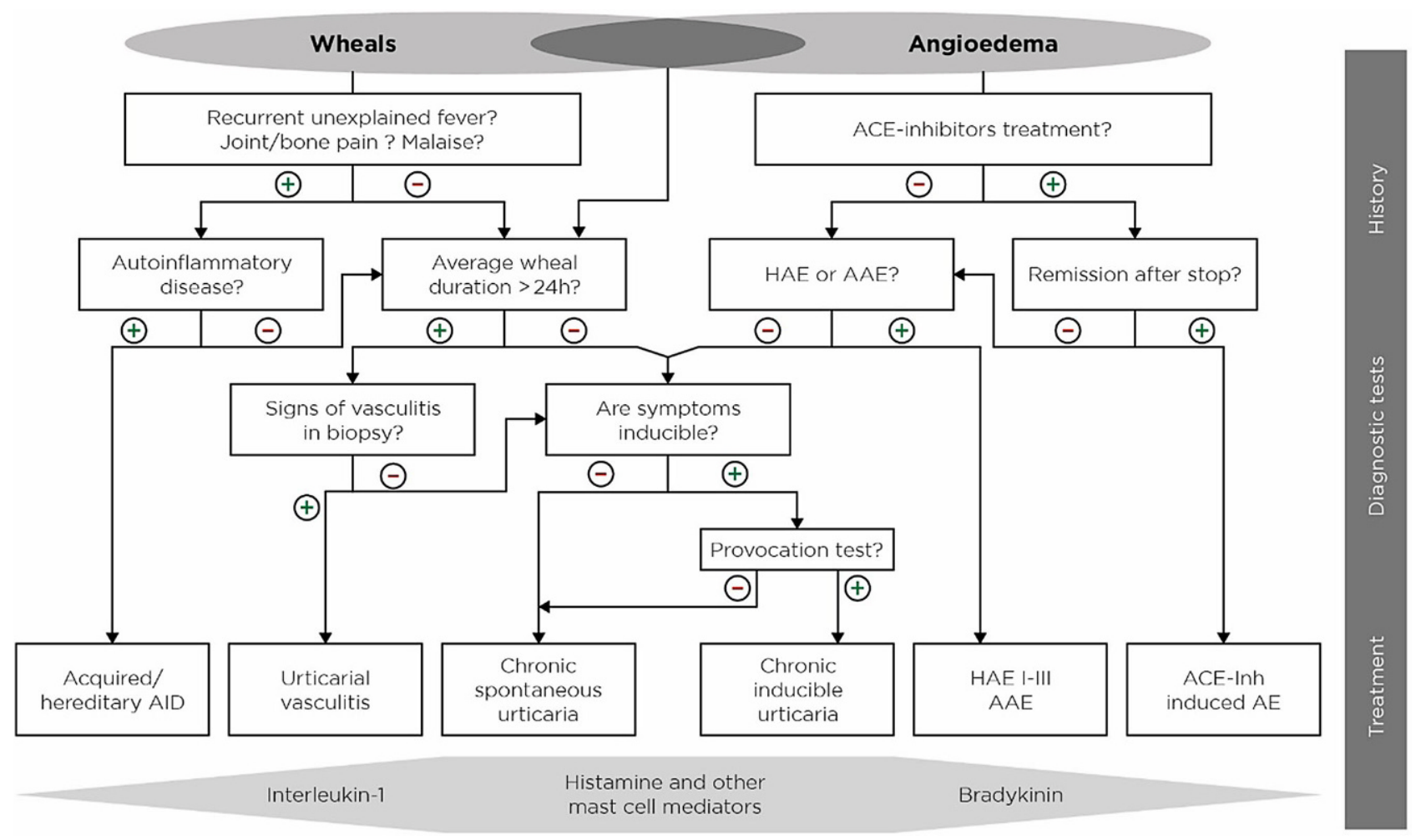

Fig. 1 Diagnostic algorithm for patients with wheals and/or angioedema. $A A E$ acquired angioedema due to $\mathrm{C} 1$-inhibitor deficiency, $A C E-I n h$ angiotensin-converting enzyme inhibitor,
$A E$ angioedema, $A I D$ auto-inflammatory disease, $H A E$ hereditary angioedema [10] 


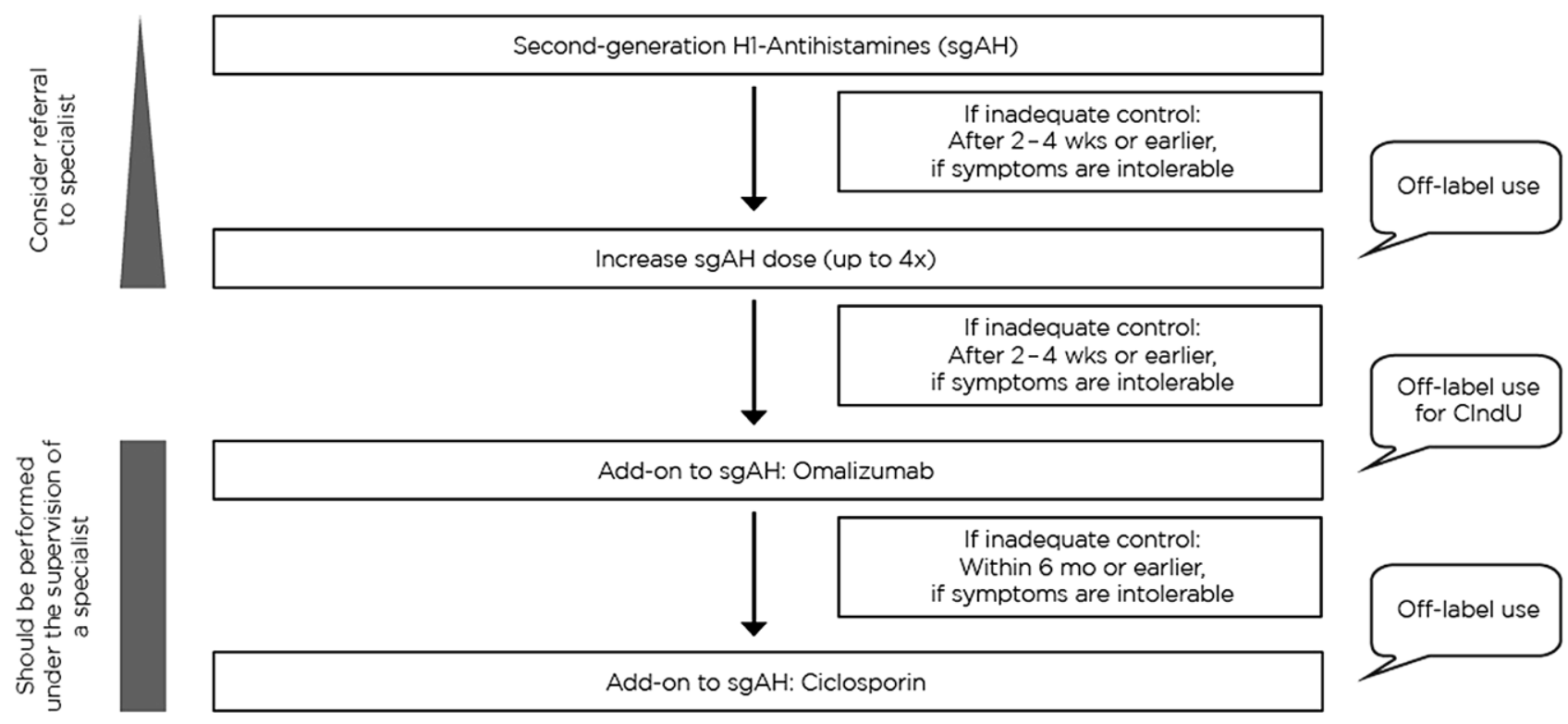

Fig. 2 Recommended therapy algorithm for the treatment of chronic urticaria. wks weeks, mo months, ClndU chronic inducible urticaria

case of light urticaria, the triggering wavelength range should be determined by means of "light stairs". In patients whose urticaria reacts to ultraviolet (UV) light, UV protection and/or UV hardening can reduce the development of urticarial complaints.

If pseudo allergy is suspected and daily symptoms are present, it is recommended that a low-pseudo allergen diet is followed for at least two to three and a maximum of four weeks. If gastrointestinal symptoms are also present, a low-histamine diet may also be advisable [19]. As yet, however, there is little evidence of the effect of diets on urticaria symptoms [10].

\section{Guideline-based therapy with $\mathrm{H} 1$ antihistamines (therapy levels 1 and 2)}

CU should always be treated in the same way, regardless of whether wheals, angioedema or both occur. Thus, the therapy of CIndU is equivalent to therapy of csU. Symptomatic therapy should be carried out according to the levels scheme recommended by the guidelines (Fig. 2; [10]).

A second-generation non-sedating $\mathrm{H} 1$ antihistamine is the first treatment of choice. If continuous intake over two to four weeks does not lead to adequate control of symptoms, the guideline recommends a higher dosage up to four times of standard dosage. This higher dosage is often more effective than the standard dosage [20], but is off-label use.

\section{Type of higher dosing}

In this situation, $2-0-2$ is the preferred treatment regimen, alternatively one tablet can be taken four times a day; however, this requires very good compliance of the patient. It is not recommended to combine different H1-antihistamines.

\section{Risk of sedation}

In case of higher dosing, second-generation $\mathrm{H} 1$ antihistamines without significant influence on road safety should be used in the approved daily dose (Table 1). Individual tolerance as well as a possible increase in sedative effects at higher doses should be considered. The patient should be informed about off-label use and possible sedation. In certain situations, e.g. in road traffic or passenger transport, there may be contraindications for off-label dosages.

\section{Cardiac risks}

Second-generation $\mathrm{H} 1$ antihistamines are generally well tolerated. A recent publication shows that they do not exhibit any signs of cardiotoxicity, even though higher doses of up to four times the standard dose are used (including bilastine and rupatadine) [23]. Therefore, in most patients, a higher dose of the standard dose is unproblematic. Possible risk factors include a hereditary long QT syndrome, the simultaneous intake of drugs that can prolong the QT time, or pre-existing heart diseases (e.g. bradycardia). These should be excluded beforehand.

\section{Guideline-based additional therapy with omalizumab (therapy level 3)}

If there is no sufficient improvement after two to four weeks of therapy with a higher-than-standard dose of second-generation $\mathrm{H} 1$ antihistamine, treatment with approved IgE antibodies such as omalizumab should 
Table 1 Road safety categorisation of $\mathrm{H} 1$ antihistamine drugs based on blood alcohol equivalent doses [21, 22]

\begin{tabular}{|c|c|c|c|}
\hline $\begin{array}{l}\text { Generation } \mathrm{H} 1 \\
\text { antihistamines }\end{array}$ & No significant effect (DE to BAC: $<0.5 \mathrm{~g} / \mathrm{l} ;<0.5$ per mil) & $\begin{array}{l}\text { II } \\
\text { Low or moderate effect (DE to BAC: } \\
0.5-0.8 \mathrm{~g} / \mathrm{l} ; 0.5-0.8 \text { per mil) }\end{array}$ & $\begin{array}{l}\text { III } \\
\text { Strong effect, potentially dangerous (DE } \\
\text { to BAC: }>0.8 \text { per mil) }\end{array}$ \\
\hline 1st Generation & - & Ketotifen & $\begin{array}{l}\text { Clemastine, diphenhydramine, pro- } \\
\text { methazine }\end{array}$ \\
\hline 2nd Generation & $\begin{array}{l}\text { Azelastine, bilastine, desloratadine, ebastine, fexofena- } \\
\text { dine, levocetirizine, loratadine, rupatadine }\end{array}$ & Cetirizine, mizolastine & - \\
\hline
\end{tabular}

Table 2 Important ICD-10 codes

\begin{tabular}{|c|c|c|c|}
\hline & ICD-10 Code & Description & Practical implementation \\
\hline \multirow[t]{4}{*}{ Urticaria } & L50.1 & Idiopathic urticaria & Acute urticaria \\
\hline & $\mathrm{L} 50.2-.6+\mathrm{L} 56.3$ & Inducible urticaria (per cause) & Inducible urticaria (coldness, warmth, etc.) \\
\hline & L50.8 & $\begin{array}{l}\text { Other urticaria } \\
\text { Chronic } \\
\text { Recurrent, periodic }\end{array}$ & To be preferred as coding for chronic spontaneous urticaria \\
\hline & L50.9 & Urticaria & Not recommended for coding \\
\hline Angioedema & T78.3 & Angioneurotic oedema/Quincke's oedema & - \\
\hline \multirow[t]{2}{*}{ Pruritus } & L29.8 & Other pruritus & - \\
\hline & L29.9 & Pruritus not specified & - \\
\hline \multirow{3}{*}{$\begin{array}{l}\text { Additional } \\
\text { codes }\end{array}$} & $\mathrm{Z72.8}$ & Highly debilitating living situation & - \\
\hline & Z51.88 & System therapy & - \\
\hline & T88.8 & Only slight improvement in symptoms & - \\
\hline
\end{tabular}

additionally be administered (Fig. 2). The same recommendations apply to children and older patients.

Omalizumab is approved for the treatment of csU from the age of 12 years but may also be considered in younger patients. Case reports in children under 12 years of age indicate that efficacy and safety do not differ between paediatric and adult patients [24, 25 ]. For the treatment of severe allergic asthma, omalizumab is approved in children as young as 6 years of age and the safety data show that it is well tolerated [26], so that off-label use in children as young as 6 years of age can be considered. This must, however, be discussed accordingly with the parents or guardians and is the sole responsibility of the attending physician.

Omalizumab treatment is also described as effective and safe in patients with CIndU [39], but it is not approved for this indication and therefore represents an individual treatment use. This applies to patients who only suffer from an inducible form and not also from csU. For patients who suffer from both CIndU and csU, therapy with omalizumab is within the label. When documenting the prescription of omalizumab, it is therefore important to ensure that both forms of CU (CIndU and csU) are well documented.

\section{Dosage and interval}

The approved dose of omalizumab in Europe is $300 \mathrm{mg}$ every four weeks as a subcutaneous injection. Other doses and interval changes have been studied [27, 28], but are not approved. In individual cases of a partial response only, an increase in dosage or a reduction of the interval can be considered. If the response is very good, a dose reduction or an extension of the interval may be eligible [29]. Any deviation from the approved scheme, however, is considered off-label use.

\section{Safety}

\section{Anaphylaxis}

The results of clinical trials show that omalizumab has a good safety profile [30]. It is important to note that omalizumab is a biological with a low rate of anaphylaxis $(0.2 \%)$. This has been shown in both, pivotal clinical trials [31-33] as well as post-marketing analyses [34, 35]. Most anaphylaxis occurred within $2 \mathrm{~h}$ of injection during the first three applications [35]. Patients with anaphylaxis in their medical history [36] due to food, drugs (especially biologicals), vaccinations, polysorbate or insect venom are at higher risk for anaphylaxis. Patients should be educated about possible symptoms of anaphylactic shock (skin reactions, effects on the respiratory tract and cardiovascular system). However, it should also be pointed out that in the case of mild reactions (e.g. increased wheals), injections can be continued without elevated anaphylaxis risk. 


\section{Malignancies}

In the phase I-III clinical trials from the asthma study programme, a numerical, but not significant imbalance in malignancies was observed between malignant tumours in patients treated with omalizumab and control patients. A causal relationship between medication and malignant neoplasms was considered unlikely due to the variety of tumour entities and the relatively short exposure [37]. Long-term data from the EXELS follow-up study show no differences regarding malignancy risk between patients with or without omalizumab therapy [38] and a pooled data analysis of 32 randomised, double-blind, placebocontrolled studies also found no association between omalizumab and an increased risk of malignancy [39]. Patients with active malignant tumours must be individually assessed for the risk/benefit of using omalizumab. There is no absolute contraindication.

\section{Combination with other biologicals}

Biologics have significantly expanded the therapeutic options for various diseases in recent years, so that their use in clinical practice is increasing. It is therefore possible that CU patients may receive another biological in addition to omalizumab due to another disease (e.g. atopic dermatitis or rheumatoid arthritis). The data situation regarding the safety and efficacy of various combinations of biologicals has so far not been sufficiently investigated. However, based on case reports and our own experience, no safety risks have been identified to date in combining omalizumab with other biologicals, such as mepolizumab, benralizumab, dupilumab or etanercept [40-43].

\section{Infections}

Infections can be triggering factors of CU. In clinical practice, the question frequently arises whether omalizumab can be used without risk in patients with infections. Pragmatically, pausing omalizumab in the case of febrile infections or systemic need for treatment with antibiotics until complete healing can be useful. In cases of a mild cold, cough, or hoarseness, omalizumab can be administered without hesitation.

\section{Practical aspects}

\section{Vaccinations under omalizumab}

Attenuated live vaccines as well as inactivated vaccines provide protection by neutralising IgG antibodies produced by B lymphocytes. No impairment of this pathway by the anti-IgE antibody omalizumab is known. As the mechanism of action of omalizumab does not cause immunosuppression, there is no contraindication to live vaccines (e.g. measles, mumps, rubella). Patients undergoing omalizumab therapy should therefore not be deprived of vaccinations. An interval of at least one week between an injection of omalizumab and plannable vaccinations is rec- ommended. Immediate necessary vaccinations (e.g. tetanus) can be given at any time.

\section{Self-administration}

Patients without a history of anaphylaxis may inject omalizumab themselves or have a caregiver inject it from the fourth use onwards if a doctor considers it appropriate. Treatment should be given for an initial period of six months, followed by a review of disease activity. If the patient fears the occurrence of anaphylaxis, the administration should be carried out again in the practice. Risk patients with anaphylactic reactions in their medical history and pregnant women are excluded from the possibility of self-administration. If patients relapse after discontinuation of omalizumab and treatment with omalizumab is renewed, this can also be done by self-administration.

Before starting the administration, the skin should be disinfected. The recommended place for self-administration is the subcutis of the abdominal wall or the side of the thigh extensor. If the injection is carried out by a caregiver, it can be injected subcutaneously into the upper arm. The individual steps of the administration as well as important information on transport and storage can be found on the package insert. Patients should be made aware that in case of intending to travel, a multilingual medical certificate confirming the necessity of carrying omalizumab in their hand luggage is required, while observing the storage conditions (e.g. on-board refrigerator or medicine cooler).

\section{Guideline-based additional therapy with ciclosporin A (therapy level 4)}

If there is no therapeutic success after six months of treatment with omalizumab, the guidelines recommend off-label use with ciclosporin A (CSA) in addition to existing therapy with $\mathrm{H} 1$ antihistamines. Recommended dosages are $4 \mathrm{mg} / \mathrm{kg}$ or less [44]. Experience shows that a therapeutic response occurs within four to eight weeks. Since adverse effects can occur more frequently during therapy, this treatment option should be reserved for therapy-resistant cases and a careful monitoring of side effects should be carried out:

- Questioning and clinical examination: hypertrichosis, gingival hyperplasia, blood pressure control, tremor, paraesthesia, gastrointestinal complaints.

- Laboratory tests: BSG, CRP, blood count including platelets, alkaline phosphatase, alanine aminotransferase, creatinine, potassium, urine test strip.

CSA should not be used in patients with impaired kidney function, untreated hypertension, untreated infections, or any form of malignancy. The patient should be informed about the off-label use of CSA, as well as the control examinations and possible side effects. Information sheets for patients and doctors 
can be downloaded (in German) from the website of the German Society for Rheumatology (https:// dgrh.de/Start/Versorgung/Therapieinformationen/ Therapieinformationsbögen.html).

\section{Systemic glucocorticoids}

In case of acute exacerbations, treatment with sufficient doses of oral systemic glucocorticoids can be given for a short period (up to a maximum of ten days) to reduce the duration and activity of the disease. A medium-high dose of prednisolone of 20-50 mg/day for a maximum of ten days is recommended. There is usually no need to taper the medications if used for three to five days $[10,44]$. Long-term treatment with systemic glucocorticoids should be avoided at all costs due to the high rate of side effects.

\section{Documentation of therapy in the clinical practice}

The therapeutic goal in the treatment of CU is to achieve complete freedom of symptoms. With the therapy options available today, this goal can be achieved for many patients. However, not all options/ doses are within the label, so that good documentation of the guideline-based therapy is of high relevance, also with regard to off-label use not being tolerated by pharmaceutical companies. The documentation must show that the CU is a serious and debilitating disease with lasting effects. This is sometimes questioned by the payers. In the best case, quality of life questionnaires should be used. If this is not possible in terms of time, anamnesis entries can also be made that reflect the severity of the suffering.

Many patients can be adequately treated with nonprescription $\mathrm{H} 1$ antihistamines. At the expense of the statutory health insurance system, these drugs for adults can only be prescribed in exceptional cases for very severe forms of CU or severe, long-lasting pruritus, which must be documented in the patient's medical file. Non-sedative antihistamines can be prescribed for patients insured by public health insurance if nonprescription second-generation $\mathrm{H} 1$ antihistamines (loratadine, desloratadine, cetirizine, levocetirizine) were proven to be ineffective or incompatible. However, this is rare and must be documented in the patient's medical file. Exceptions to this rule apply for example to children up to the age of 12 . If an overthe-counter (OTC) therapy is not listed in the medical file, as OTC prescriptions do not have to appear there, another form of proof that such a therapy attempt has been made is required.

If the standard dose of second-generation $\mathrm{Hl}$ antihistamines does not lead to symptom control, a subsequent fourfold dosage of second-generation $\mathrm{H} 1$ antihistamines follows the guidelines and is safe according to current knowledge. However, patients should be advised that higher doses of second-generation $\mathrm{Hl}$ antihistamines as well as treatment with
CSA are equivalent to off-label use. The corresponding risk disclosure and the information of possible non-reimbursement by statutory health insurances must be carefully documented and should best be countersigned by the patient. Privately insured patients should ask their health insurance company about a possible reimbursement before starting therapy. Lack of therapy successes should best be documented with scores. The UCT can be carried out in an outpatient setting with little effort. The UAS7 in the form of an urticaria diary can also be very helpful in difficult disease courses.

The csU is not uniquely codable, but the coding can be supplemented via a text box within the software. Important codes for patients with urticaria are shown in Table 2.

\section{Urticaria and family planning}

In general, patients with CU can have children. Therapy options are available that can also be used during pregnancy. The therapeutic goal of freedom of symptoms also applies during pregnancy. Pregnant women with uncontrolled CU should be referred to a specialised centre.

Although $\mathrm{H}$ antihistamines are not approved for use during pregnancy, experience (especially with loratadine and cetirizine) does not indicate an increased risk of malformation. These two substances can preferably be given in a once daily dosage (https://www. embryotox.de/). The desire to have children is not a reason to stop or omit therapy with loratadine or cetirizine if this results in good symptom control of CU. First-generation $\mathrm{H} 1$ antihistamines, on the other hand, are not recommended even at night due to placental passage and the disruption of REM (rapid eye movement) sleep. There have been no studies on the safety of up dosing of second-generation H1 antihistamines in pregnant women.

For omalizumab, the EXPECT pregnancy registry found no evidence of a pregnancy-induced risk of congenital anomalies or thrombocytopenia in patients with asthma (230 documented pregnancies) [45]. The guideline also describes the use of omalizumab in pregnancy as safe [10]. If there is a clinical need, which may be caused by the patient's exposure to uncontrolled CU including sleep loss, the use of omalizumab during pregnancy may be considered after appropriate risk assessment and patient education. However, self-administration is not recommended for these patients.

\section{Summary and conclusion for clinical practice}

- With the aid of thorough anamnesis, physical examination, and basic laboratory diagnostics, a diagnosis can be made in a short time. 
- For the anamnesis, a short basic form or the more detailed CURE patient forms for initial and followup presentation are suitable.

- An extended diagnostic evaluation is only indicated in a few cases and should always be carried out in parallel with an effective therapy.

- Angioedema are often underdiagnosed in patients with csU, although they can be part of csU regardless of the presence of wheals and can have a negative impact on quality of life and daily activities.

- A csU is therefore always treated in the same way, independent of the presence of wheals angioedema, or both.

- The treatment of choice for csU is a second-generation $\mathrm{H} 1$ antihistamine. Higher doses, up to a maximum of four times the standard dose, are often more effective but are off-label use and are not tolerated by all patients. In most patients, however, a higher dosage of the standard dose is unproblematic if potential risk factors or comedication are considered (no signs of cardiotoxicity).

- If there is no adequate improvement after 2-4 weeks with a second-generation $\mathrm{Hl}$ antihistamine in the standard dosage or, if necessary, after higher dosages, patients with csU should additionally be given omalizumab.

- The therapy of CIndU is equivalent to the therapy of csU. For patients who suffer from both CIndU and csU, therapy with omalizumab is within the label.

- Omalizumab has a good safety profile with a low anaphylaxis rate.

- In cases of mild rhinitis, cough, or hoarseness, omalizumab can be administered without concern.

- Both inactivated and live vaccines can be administered under omalizumab therapy.

- Patients with no known anaphylaxis in their medical history can inject omalizumab themselves or have a caregiver inject it from the fourth application onwards (except pregnant women).

- If there is no therapeutic success after 6 months of treatment with omalizumab, off-label use with CSA in addition to existing therapy with $\mathrm{Hl}$ antihistamines is recommended by the guidelines instead.

- In cases of acute exacerbations, treatment with medium-dose oral systemic glucocorticoids can be given for a short period (up to 10 days maximum) to reduce disease duration and activity.

- If clinically necessary, the use of second-generation $\mathrm{H1}$ antihistamines (loratadine, cetirizine) and omalizumab during pregnancy may be considered (offlabel).

- Good documentation and education of patients about off-label use is of great importance (the responsibility lies with the treating physician).

Funding The authors would like to thank Dr Maren Klug (KW MEDIPOINT) for editorial support. This was funded by Novartis Pharma $\mathrm{GmbH}$ in accordance with guidelines on Good Publication Practice (GPP3; http://www.ismpp.org/ gpp3). The preparation of this publication was financially supported by Novartis Pharma $\mathrm{GmbH}$. The authors are solely responsible for the contents.

Funding Open Access funding enabled and organized by Projekt DEAL

Conflict of interest A. Bauer received fees for lectures and advisory boards of Novartis, Biofrontera, Shire/Takeda and Leo Pharma as well as research support from Novartis during the last three years. She has been involved in clinical trials with drugs from Novartis, Lilly, Regeneron, Sanofi, Leo Pharma, and Genetec. H. Dickel received fees from Novartis for lectures and advisory boards. He is also involved as PI in clinical studies with preparations from Novartis and Leo Pharma. T. Jakob has received fees for lectures and advisory boards from Novartis, ALK-Abéllo, Allergopharma, Bencard, Thermo Fischer and Celegene, as well as research support from Novartis and ALK-Abéllo. He is also a member of the extended board of the German Society for Allergology and Clinical Immunology and editor of the Allergo Journal and Allergo Journal International. A. Kleinheinz received financial support from Novartis, LEO, Sanofi, Galderma, Abbvie, Janssen, Medac, Almirall, Amgen, Celgene, Genentech, GSK, Lilly, Parexel, Pfizer and proinnovera as a speaker and for participation in clinicial studies. U. Lippert received fees for lectures or advisory boards lasting several hours in the last three years from Novartis, Shire/Takeda, ALK-Abéllo, Meda Pharma GmbH and Sanofi. She received research grants and consulting fees from Novartis and travel expenses from Novartis and Shire/Takeda. She has also been involved as LKP and PI in clinical trials with preparations from Regeneron, Sanofi and Novartis. M. Metz received fees as speaker and/or consultant for Amgen, Aralez, argenx, Moxie, Novartis, Roche, Sanofi and Uriach. S. Schliemann is an investigator in studies by Novartis Pharma GmbH, Sanofi GmbH, Eli Lilly and Company Limited, LEO Pharma GmbH, Pierre Fabre DermoCosmetique, has received lecture fees from Novartis Pharma $\mathrm{GmbH}$, Sanofi GmbH, LEO Pharma GmbH and HAL Allergy $\mathrm{GmbH}$ and research support from Novartis Pharma GmbH. U. Schwichtenberg: AbbVie Deutschland GmbH, Almirall Hermal GmbH, Amgen, Beiersdorf Derma Medical GmbH, Celgene $\mathrm{GmbH}$, Dermapharm $\mathrm{GmbH}$, Janssen Cilag GmbH, Johnson \& Johnson GmbH, LEO Pharma GmbH, L'Oréal GmbH, MEDA Pharma GmbH, Merz Pharmaceuticals GmbH, MSD SHARP \& DOHME GmbH, Novartis Pharma GmbH, and Pfizer $\mathrm{GmbH}$, Sanofi-Aventis Deutschland $\mathrm{GmbH}$-fees for lectures, advisory boards, study participation, Medical Project Design GmbH Shareholder and Managing Director, Professional Association of German Dermatologists Bremen State Chairman, Association of Statutory Health Insurance Physicians Bremen Member of the Assembly of Representatives and Chairman of the Honorarium Distribution ScaleCommittee, Member of the Finance Committee. P. Staubach is or recently was a speaker and/or advisor for and/or has received research funding from AbbVie, Allergika, Almirall, Amgen, Beiersdorf, Biocryst, Biogen Idec, BMS, Boehringer-Ingelheim, Celgene, CSL-Behring, Eli-Lilly, Galderma, Hexal, Janssen, Klosterfrau, LEO-Pharma, LETI-Pharma, L'Oreal, Novartis, Octapharma, Pfizer, Pflüger, Pharming, Regeneron, Shire, Takeda, Regeneron, Sanofi-Genzyme und UCB Pharma. E. Valesky has received honours for lectures and consultancy work for the companies Novartis, Bencard, Alk-Abello, JUZO, Kreussler and Sigvaris over the last three years. N. Wagner received fees for lectures or advisory boards lasting several hours in the last three years from Novartis, Shire/Takeda, ALK-Abéllo, Allergopharma, Abbvie, as well as research support from Novartis. In addition, she was involved as PI in clinical trials with Novartis products. 
B. Wedi received fees for lectures or advisory boards lasting several hours over the last three years from Novartis, Shire/ Takeda, ALK-Abéllo, Bencard, CSL-Behring, HAL-Allergy and Leo Pharma as well as research support from Shire/Takeda. She has also been involved as LKP or PI in clinical trials with Novartis products. M. Maurer is or recently was a speaker and/or advisor for and/or has received research funding from Allakos, Amgen, Aralez, ArgenX, AstraZeneca, Celldex, Centogene, CSL Behring, FAES, Genentech, GIInnovation, Innate Pharma, Kyowa Kirin, Leo Pharma, Lilly, Menarini, Moxie, Novartis, Roche, Sanofi/Regeneron, Third HarmonicBio, UCB, and Uriach.

Open Access This article is licensed under a Creative Commons Attribution 4.0 International License, which permits use, sharing, adaptation, distribution and reproduction in any medium or format, as long as you give appropriate credit to the original author(s) and the source, provide a link to the Creative Commons licence, and indicate if changes were made. The images or other third party material in this article are included in the article's Creative Commons licence, unless indicated otherwise in a credit line to the material. If material is not included in the article's Creative Commons licence and your intended use is not permitted by statutory regulation or exceeds the permitted use, you will need to obtain permission directly from the copyright holder. To view a copy of this licence, visit http://creativecommons.org/licenses/by/4.0/.

\section{References}

1. Maurer M, Abuzakouk M, Bérard F, Canonica W, Oude Elberink H, Giménez-Arnau A, et al. The burden of chronic spontaneous urticaria is substantial: real-world evidence from ASSURE-CSU. Allergy. 2017;72:2005-16.

2. Maurer M, Staubach P, Raap U, Richter-Huhn G, BaierEbert M, Chapman-Rothe N. ATTENTUS, a German online survey of patients with chronic urticaria highlighting the burden of disease, unmet needs and real-life clinical practice. Br J Dermatol. 2016;174:892-4.

3. Mann C, Dreher M, WeeßHG, Staubach P. Sleep disturbance in patients with urticaria and atopic dermatitis: an underestimated burden. Acta Derm Venereol. 2020;100:adv73.

4. Maurer M, Raap U, Staubach P, Richter-Huhn G, Bauer A, Oppel EM, et al. Antihistamine-resistant chronic spontaneous urticaria: 1-year datafrom theAWAREstudy. Clin Exp Allergy. 2019;49:655-62.

5. Maurer M, RaapU, Staubach P, Richter-Huhn G, BaeumerD, Chapman-Rothe N, editors. Real-world evidence from the AWARE study in Germany highlights the need for greater awareness of guideline recommendations for the treatment of patients with $\mathrm{H} 1$-antihistamine-refractory chronic spontaneous urticaria. 50th DDG-Tagung; Berlin. 2019.

6. Teach SJ, Gill MA, Togias A, Sorkness CA, Arbes SJ Jr., CalatroniA, etal. Preseasonal treatmentwitheitheromalizumab or an inhaled corticosteroid boost to prevent fall asthma exacerbations. JAllergy Clin Immunol. 2015;136:1476-85.

7. Esquivel A, Busse WW, Calatroni A, Togias AG, Grindle KG, Bochkov YA, et al. Effects of omalizumab on rhinovirus infections, illnesses, and exacerbations of asthma. Am J Respir CritCare Med. 2017;196:985-92.

8. Lommatzsch M, Stoll P, Virchow JC. COVID-19 in a patient with severe asthma treated with Omalizumab. Allergy. 2020;75:2705-8.

9. Klimek L, Pfaar O, Worm M, Eiwegger T, Hagemann J, Ollert M, et al. Anwendung von Biologika bei aller- gischen und Typ-2-entzündlichen Erkrankungen in der Covid-19-Pandemie ${ }^{\mathrm{a}, \mathrm{b}, \mathrm{c}}$ : Positionspapier des Ärzteverbands Deutscher Allergologen (AeDA)(A), der Deutschen Gesellschaft für Allergologie und Klinische Immunologie (DGAKI)(B), der Gesellschaft für Pädiatrische Allergologie und Umweltmedizin (GPA)(C), der Österreichischen Gesellschaft für Allergologie und Immunologie (ÖGAI)(D), der Luxemburgischen Gesellschaft für Allergologie und Immunologie (LGAI)(E), der Österreichischen Gesellschaftfür Pneumologie (ÖGP) (F) in co-operation mit der deutschen, österreichischen und schweizerischen ARIA-Gruppe (G) und der Europäischen Akademie für Allergologie und KlinischeImmunologie (EAACI) (H). Allergo J.2020;29(4):14-27.

10. Zuberbier T, Aberer W, Asero R, Latiff AA, Baker D, BallmerWeber B, et al. EAACI/GA²LEN/EDF/WAO-Leitlinie für die Defnition, Klassifkation, Diagnose und das Management der Urtikaria - konsentierte, deutschsprachige Übersetzung. Allergo J. 2018;27:41-69.

11. Sussman G, Abuzakouk M, Bérard F, Canonica W, Oude Elberink H, Giménez-Arnau A, et al. Angioedema in chronic spontaneous urticaria is underdiagnosed and has a substantial impact: analyses from ASSURE-CSU. Allergy. 2018;73:1724-34.

12. Verneuil L, Leconte C, Ballet JJ, Coffin C, Laroche D, Izard JP, et al. Association between chronic urticaria and thyroid autoimmunity: a prospective study involving 99 patients. Dermatology. 2004;208:98-103.

13. Konstantinou GN, Konstantinou GN. Psychiatric comorbidity in chronic urticaria patients: a systematic review and meta-analysis. Clin Transl Allergy. 2019;9:42.

14. Hawro T, Ohanyan T, Schoepke N, Metz M, Peveling-Oberhag A, Staubach P, et al. The urticaria activity score-validity, reliability, and responsiveness. J Allergy Clin Immunol Pract. 2018;6:1185-1190.e1.

15. Weller K, GroffikA, Magerl M, Tohme N, Martus P, Krause K, et al. Development, validation, and initial results of the AngioedemaActivity Score. Allergy. 2013;68:1185-92.

16. Weller K, Groffik A, Church MK, Hawro T, Krause K, Metz M, et al. Development and validation of the urticaria control test: a patient-reported outcome instrument for assessing urticaria control. J Allergy Clin Immunol. 2014;133:1365-1372,72.e1-6.

17. Młynek A, Magerl M, Hanna M, Lhachimi S, Baiardini I, Canonica GW, et al. The German version of the Chronic Urticaria Quality-of-Life Questionnaire: factor analysis, validation, and initial clinical findings. Allergy. 2009;64:927-36.

18. Weller K, Groffik A, Magerl M, Tohme N, Martus P, Krause K, et al. Development and construct validation of the angioedema quality of life questionnaire. Allergy. 2012;67:1289-98.

19. Wagner N, Dirk D, Peveling-Oberhag A, Reese I, RadyPizarro U, Mitzel H, et al. A Popular myth-low-histamine diet improves chronic spontaneous urticaria-fact or fiction? J Eur Acad Dermatol Venereol. 2017;31:650-5.

20. Guillén-Aguinaga S, Jáuregui Presa I, Aguinaga-Ontoso E, Guillén-Grima F, Ferrer M. Updosing nonsedating antihistamines in patients with chronic spontaneous urticaria: a systematic review and meta-analysis. $\mathrm{Br} J$ Dermatol. 2016;175:1153-65.

21. Verster JC, Mets MA. Psychoactive medication and traffic safety. Int JEnviron Res Public Health. 2009;6:1041-54.

22. ICADTS. Categorization system for medicinal drugs affecting driving performance 2007.. http://www.icadts.nl/ medicinal.html. Accessed 17 June 2020.

23. Cataldi M, Maurer M, Taglialatela M, Church MK. Cardiac safety of second-generation $\mathrm{H}(1)$-antihistamines when up- 
dosed in chronic spontaneous urticaria. Clin Exp Allergy. 2019;49:1615-23.

24. Netchiporouk E, Nguyen $\mathrm{CH}$, Thuraisingham T, Jafarian F, Maurer M, Ben-Shoshan M. Management of pediatric chronic spontaneous and physical urticaria patients with omalizumab: case series. Pediatr Allergy Immunol. 2015;26:585-8.

25. Ossorio-García L, Jiménez-Gallo D, Albarrán-Planelles C, Arjona-Aguilera C, Linares-Barrios M. Chronic spontaneous urticaria in an 8-year-old girl treated with omalizumab. Clin Exp Dermatol. 2016;41:929-30.

26. Berger W, Gupta N, McAlary M, Fowler-Taylor A. Evaluation of long-term safety of the anti-IgE antibody, omalizumab, in children with allergic asthma. Ann Allergy Asthma Immunol. 2003;91:182-8.

27. Bernstein JA, Kavati A, Tharp MD, Ortiz B, MacDonald K, Denhaerynck K, et al. Effectiveness of omalizumab in adolescent and adult patients with chronic idiopathic/ spontaneous urticaria: a systematic review of 'real-world' evidence. Expert Opin Biol Ther. 2018;18:425-48.

28. Metz M, Vadasz Z, Kocatürk E, Giménez-Arnau AM. Omalizumab updosing in chronic spontaneous urticaria: an overview of real-world evidence. Clin RevAllergy Immunol. 2020;:59:38-45

29. Türk M, Carneiro-Leão L, Kolkhir P, Bonnekoh $\mathrm{H}$, Buttgereit T, Maurer M. How to treat patients with chronic spontaneous urticaria with omalizumab: Questions and answers. J Allergy Clin Immunol Pract. 2020;8:113-24.

30. Zhao ZT, Ji CM, Yu WJ, Meng L, Hawro T, Wei JF, et al. Omalizumab for the treatment of chronic spontaneous urticaria: a meta-analysis of randomized clinical trials. JAllergy Clin Immunol. 2016;137:1742-1750.e4.

31. Maurer M, Rosén K, Hsieh HJ, Saini S, Grattan C, Gimenéz-Arnau A, et al. Omalizumab for the treatment of chronic idiopathic or spontaneous urticaria. N Engl J Med. 2013;368:924-35.

32. Saini SS, Bindslev-Jensen C, Maurer M, Grob JJ, Bülbül Baskan E, Bradley MS, et al. Efficacy and safety of omalizumab in patients with chronic idiopathic/spontaneous urticaria who remain symptomatic on $\mathrm{H} 1$ antihistamines: a randomized, placebo-controlled study. J Invest Dermatol. 2015;135:67-75.

33. KaplanA, LedfordD, AshbyM, CanvinJ,ZazzaliJL, ConnerE, et al. Omalizumab in patients with symptomatic chronic idiopathic/spontaneous urticaria despite standard combination therapy. J Allergy Clin Immunol. 2013;132:101-9.

34. Cox L, Platts-Mills TA, Finegold I, Schwartz LB, Simons FE, Wallace DV. American Academy of Allergy, Asthma \& Im-
munology/American College of Allergy, Asthma and Immunology Joint Task Force Report on omalizumab-associated anaphylaxis. JAllergy Clin Immunol. 2007;120:1373-7.

35. Cox L, Lieberman P, Wallace D, Simons FE, Finegold I, Platts-Mills T, et al. American Academy of Allergy, Asthma \& Immunology/American College of Allergy, Asthma \& Immunology Omalizumab-Associated Anaphylaxis Joint Task Force follow-up report. J Allergy Clin Immunol. 2011;128:210-2.

36. Lieberman PL, Jones I, Rajwanshi R, Rosén K, Umetsu DT. Anaphylaxis associated with omalizumab administration: risk factors and patient characteristics. J Allergy Clin Immunol. 2017;140:1734-1736.e4.

37. Fernandez C, Busse W, Reisner C, Gupta N, editors. Clinical data do not suggest a causal relationship between omalizumab therapy and cancer. Proc Am Thorac Soc; 2005. p. A359

38. Long A, Rahmaoui A, Rothman KJ, Guinan E, Eisner M, Bradley MS, et al. Incidence of malignancy in patients with moderate-to-severe asthma treated with or without omalizumab. JAllergy Clin Immunol. 2014;134:560-567.e4.

39. Busse W, Buhl R, Fernandez Vidaurre C, Blogg M, Zhu J, Eisner MD, et al. Omalizumab and the risk of malignancy: results from a pooled analysis. J Allergy Clin Immunol. 2012;129:983-989.e6.

40. Altman MC, Lenington J, Bronson S, Ayars AG. Combination omalizumab and mepolizumab therapy for refractory allergic bronchopulmonary aspergillosis. J Allergy Clin Immunol Pract. 2017;5:1137-9.

41. Dedaj R, Unsel L. Case study: a combination of mepolizumab and omaluzimab injections for severe asthma. JAsthma. 2019;56:473-4.

42. Ortega G, Tongchinsub P, Carr T. Combination biologic therapy for severe persistent asthma. Ann Allergy Asthma Immunol. 2019;123:309-11.

43. Ghazanfar MN, Thomsen SF. Combined treatment with omalizumab and etanercept in a patient with chronic spontaneous urticaria and rheumatoid arthritis. J Dermatolog Treat. 2019;30:387-8.

44. Termeer C, Staubach P, Kurzen H, Strömer K, Ostendorf R, Maurer M.Chronic spontaneous urticaria-a management pathway for patients with chronic spontaneous urticaria. JDtsch Dermatol Ges. 2015;13:419-28.

45. Namazy JA, Blais L, Andrews EB, Scheuerle AE, Cabana MD, Thorp JM, et al. Pregnancy outcomes in the omalizumab pregnancy registry and a disease-matched comparator cohort. JAllergy Clin Immunol. 2020;145:528-536.e1. 